University of Nebraska - Lincoln

DigitalCommons@University of Nebraska - Lincoln

Ralph Skomski Publications

Research Papers in Physics and Astronomy

2011

Spin correlations and Kondo effect in a strong ferromagnet

\author{
Parashu Kharel \\ University of Nebraska-Lincoln, pkharel2@unl.edu \\ Ralph Skomski \\ University of Nebraska-Lincoln, rskomski2@unl.edu \\ Pavel Lukashev \\ University of Nebraska-Lincoln, pavel.lukashev@uni.edu \\ Renat F. Sabirianov \\ University of Nebraska at Omaha, rsabirianov@mail.unomaha.edu \\ David J. Sellmyer \\ University of Nebraska-Lincoln, dsellmyer@unl.edu
}

Follow this and additional works at: https://digitalcommons.unl.edu/physicsskomski

Kharel, Parashu; Skomski, Ralph; Lukashev, Pavel; Sabirianov, Renat F.; and Sellmyer, David J., "Spin correlations and Kondo effect in a strong ferromagnet" (2011). Ralph Skomski Publications. 87. https://digitalcommons.unl.edu/physicsskomski/87

This Article is brought to you for free and open access by the Research Papers in Physics and Astronomy at DigitalCommons@University of Nebraska - Lincoln. It has been accepted for inclusion in Ralph Skomski Publications by an authorized administrator of DigitalCommons@University of Nebraska - Lincoln. 


\title{
Spin correlations and Kondo effect in a strong ferromagnet
}

\author{
P. Kharel, ${ }^{1,2}$ R. Skomski, ${ }^{1,2}$ P. Lukashev, ${ }^{1,2}$ R. Sabirianov, ${ }^{1,3}$ and D. J. Sellmyer ${ }^{1,2}$ \\ ${ }^{1}$ Nebraska Center for Materials and Nanoscience, University of Nebraska, Lincoln, Nebraska 68588, USA \\ ${ }^{2}$ Department of Physics and Astronomy, University of Nebraska, Lincoln, Nebraska 68588, USA \\ ${ }^{3}$ Department of Physics and Astronomy, University of Nebraska, Omaha, Nebraska 68182, USA
}

(Received 28 April 2011; published 29 July 2011)

\begin{abstract}
The spin structure and electron-transport behavior of Pt-substituted MnBi thin films have been investigated. The electrical resistivity of these ferromagnetic films shows an unusual low-temperature resistance minimum or Kondo effect accompanied by positive magnetoresistance. First-principles calculations show that Mn atoms displaced to the bipyramidal interstitial sites are antiferromagnetically coupled to the $\mathrm{Mn}$ atoms on their regular lattice sites. We explain the observed Kondo effect and the positive magnetoresistance as the consequences of local spin correlations involving Mn atoms displaced to interstitial sites by Pt doping.
\end{abstract}

DOI: 10.1103/PhysRevB.84.014431

PACS number(s): 75.20.Hr, 72.10.Fk, 72.15.Qm, 73.50.-h

\section{INTRODUCTION}

The Kondo effect is one of the most widely studied phenomena in condensed-matter physics. Its most famous manifestation is a resistance minimum as a function of temperature, ${ }^{1,2}$ but the effect has recently received renewed attention due to developments in nanoscience. ${ }^{3-5}$ In the classical Kondo effect, ${ }^{1}$ the resistance minimum is caused by spin-flip scattering of conduction electrons by dilute magnetic impurities. In magnetically ordered Kondo lattices (heavyfermion compounds), relatively weak interatomic exchange and Kondo spin-flip scattering can operate simultaneously. ${ }^{6-10}$ However, in strong ferromagnets with large exchange interaction and ordering temperatures above room temperature, the Kondo effect is suppressed, because it fixes the local spin directions. Here, we present our investigation of how unusual spin correlations can create a resistance minimum consistent with the Kondo effect in such a material, Pt-substituted MnBi.

$\mathrm{MnBi}$ is an intriguing ferromagnetic material, both magnetically and structurally. Manganese alloys tend to exhibit antiferromagnetic (AFM) order, because they have nearly halffilled $3 d$ bands, but $\mathrm{MnBi}$ is one of the few ferromagnetic (FM) manganese compounds discovered so far, and it can be used as a permanent-magnet material. ${ }^{11}$ Other interesting magnetic properties are an extraordinarily large Kerr rotation, ${ }^{12,13}$ a Curie temperature well above room temperature, ${ }^{14}$ a large perpendicular room-temperature anisotropy in thin films, ${ }^{15}$ and a high coercivity that increases with temperature. ${ }^{16}$ The situation is further complicated by the involvement of ordered and disordered phases. The low-temperature phase (LTP) of $\mathrm{MnBi}$ is ferromagnetic and has the hexagonal NiAs structure shown in Fig. 1(a). It consists of alternating $\mathrm{Mn}$ and $\mathrm{Bi}$ layers with ABAC stacking (as contrasted to the $\mathrm{ABC}$ and $\mathrm{AB}$ stackings of the fcc and hep structures) and exhibits large trigonal-bipyramidal interstitial sites, which may be occupied by dopant or Mn atoms. With increasing temperature, the material remains ferromagnetic up to $628 \mathrm{~K}$ and then undergoes a coupled structural and magnetic phase transition to a paramagnetic high-temperature phase (HTP). ${ }^{13}$ The hightemperature phase is a disordered NiAs phase where $10-15 \%$ of the large bipyramidal interstitial sites are occupied by Mn atoms. ${ }^{17,18}$ Rapid cooling of HTP MnBi yields a quenched high-temperature phase (QHTP), which is ferromagnetic with a Curie temperature of about $440 \mathrm{~K}$.

\section{MATERIALS AND METHODS}

Our Pt-doped MnBi thin films were prepared using an e-beam evaporation system by sequential evaporation of $\mathrm{Bi}$, $\mathrm{Mn}$, and Pt onto a glass substrate. To form the alloys, $\mathrm{Bi} / \mathrm{Mn}$ bilayer and $\mathrm{Bi} / \mathrm{Mn} / \mathrm{Pt}$ trilayer samples were deposited at a substrate temperature of $125^{\circ} \mathrm{C}$ and then annealed in situ at $375^{\circ} \mathrm{C}$ for $1 \mathrm{~h} .{ }^{19}$ Data were collected on samples having stoichiometries of $\mathrm{Mn}_{55-x} \mathrm{Pt}_{x} \mathrm{Bi}_{45}(x=0,1.5,3,4,4.5)$ and a thickness of about $32 \mathrm{~nm}$. Figure 1(b) shows the X-ray diffraction patterns for the $\mathrm{Mn}_{55-x} \mathrm{Pt}_{x} \mathrm{Bi}_{45}$ thin films with $x=0$ and $x=4.5$. The polycrystalline films have a hexagonal NiAs crystal structure and are highly textured. The strong diffraction peaks from (002) and (004) planes indicate that the films have a preferred $c$-axis orientation. Since roomtemperature $\mathrm{MnBi}$ exhibits an easy magnetization direction along the $c$ axis, this corresponds to a magnetocrystalline easy axis perpendicular to the film plane. There is no evidence for alloy secondary-phase formation and Mn clustering, although reflections from unreacted $\mathrm{Bi}$ have been detected.

\section{RESULTS AND DISCUSSION}

The magnetic hysteresis loops were recorded in the outof-plane geometry where the magnetic field was applied perpendicular to the film plane. Figure 2(a) compares the out-of plane room-temperature hysteresis loop of the film with the highest Pt content $(x=4.5)$ with that of Pt-free $\mathrm{Mn}_{55} \mathrm{Bi}_{45}$. All $\mathrm{Mn}_{55-x} \mathrm{Pt}_{x} \mathrm{Bi}_{45}$ thin films exhibit nearly rectangular hysteresis loops, but the saturation magnetization $M_{s}$ and coercivity $H_{c}$ change significantly as a function of $\mathrm{Pt}$ concentration. The magnetization decreases from $506 \mathrm{emu} / \mathrm{cm}^{3}(x=0)$ to $311 \mathrm{emu} / \mathrm{cm}^{3}(x=4.5)$ and coercivity increases from $5.7 \mathrm{kOe}$ $(x=0)$ to $17.7 \mathrm{kOe}(x=4.5)$. It is interesting to note that the magnetization decreases much faster than predicted by simple dilution from the fraction of Pt atoms; that is, a $40 \%$ drop in magnetization is caused by only $4.5 \% \mathrm{Pt}$. The disproportional magnetization decrease suggests that the $\mathrm{Pt}$ enhances the interstitial occupancy of the $\mathrm{Mn}$ atoms, which are coupled 

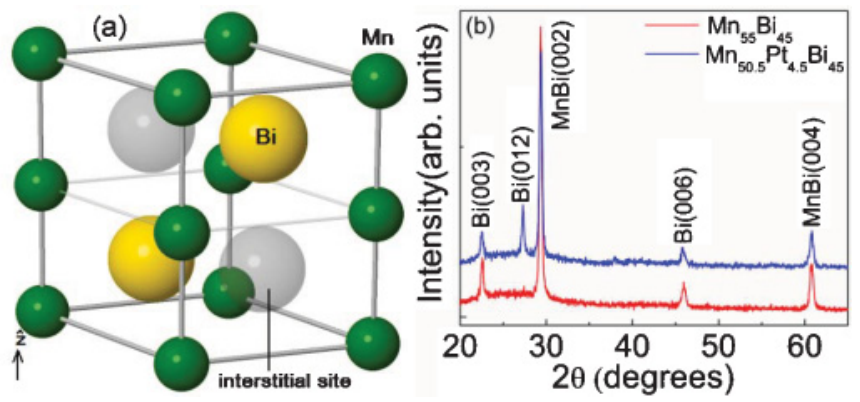

FIG. 1. (Color online) (a) Crystal structure of MnBi. Pt goes onto the regular Mn sites in the hexagonal NiAs structure, which displaces some of the $\mathrm{Mn}$ atoms to the interstitial sites. The $c$ axis is parallel to $\hat{z}$. (b) X-ray diffraction patterns for $\mathrm{Mn}_{55} \mathrm{Bi}_{45}$ and $\mathrm{Mn}_{50.5} \mathrm{Pt}_{4.5} \mathrm{Bi}_{45}$ thin films. The baseline of the $\mathrm{Mn}_{50.5} \mathrm{Pt}_{4.5} \mathrm{Bi}_{45}$ pattern is shifted for clarity.

antiferromagnetically to the original $\mathrm{Mn}$ sublattice. In fact, this is consistent with neutron diffraction data that indicate interstitial manganese tends to couple antiferromagnetically to its Mn neighbors on the regular lattice sites. ${ }^{20}$ This is a natural consequence of the predominantly antiferromagnetic exchange of $\mathrm{Mn}$ atoms, which need specific requirements, such as the regular lattice occupancy in $\mathrm{MnBi}$, to become ferromagnetic.

Support for the interstitial mechanism of magnetization reduction due to $\mathrm{Pt}$ addition is provided by first-principles calculations that we performed using the projector augmentedwave method and its implementation in the Vienna ab initio simulation package (VASP) code within the Perdew-BurkeErnzerhof generalized gradient approximation. ${ }^{21}$ We have implemented full atomic relaxation with total forces of less than $0.005 \mathrm{eV} / \AA$ after relaxation and have examined both the FM and AFM couplings between interstitial and regular Mn spins. Four types of supercells are considered, each containing 32 atoms: (i) ordered $\mathrm{MnBi}\left(\mathrm{Mn}_{16} \mathrm{Bi}_{16}\right)$, corresponding to the LTP; (ii) disordered $\mathrm{MnBi}\left(\mathrm{Mn}_{16} \mathrm{Bi}_{16}\right)$, roughly corresponding to the QHTP; (iii) solid solution of Pt in ordered $\mathrm{MnBi}$, where one $\mathrm{Mn}$ atom in each cell is replaced by $\mathrm{Pt}\left(\mathrm{Mn}_{15} \mathrm{PtBi}_{16}\right)$; and (iv) $\mathrm{Pt}$ in disordered $\mathrm{MnBi}$, where the $\mathrm{Pt}$ displaces one $\mathrm{Mn}$
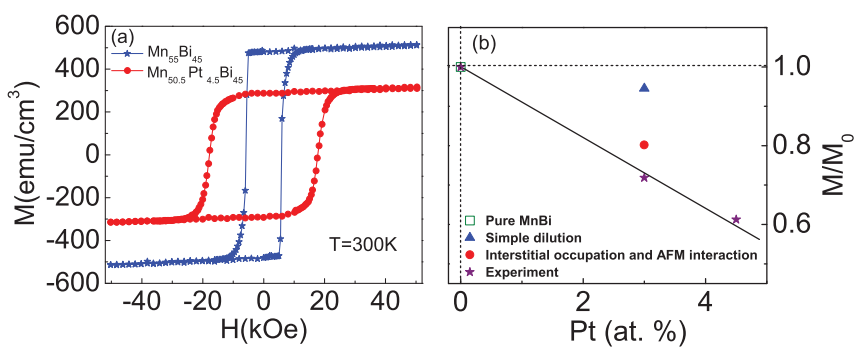

FIG. 2. (Color online) (a) $M(H)$ hysteresis loops for the $\mathrm{Mn}_{55} \mathrm{Bi}_{45}$ and $\mathrm{Mn}_{50.5} \mathrm{Pt}_{4.5} \mathrm{Bi}_{45}$ films recorded at room temperature with magnetic field perpendicular to the film plane. $M(H)$ loops are almost rectangular for both the samples. (b) Normalized magnetization as a function of Pt concentration. Solid triangles and circles represent the computational values of magnetization for 3\% Pt-doped samples and the experimentally observed values of magnetizations are marked by stars. The solid black line is a visual guide.
TABLE I. The average magnetic moment per Mn atom $\langle m\rangle$ $\left(\mu_{B} / \mathrm{Mn}\right)$. The first two columns show the values of $\langle m\rangle$ for $\mathrm{Mn}_{16} \mathrm{Bi}_{16}$ and $\mathrm{Mn}_{15} \mathrm{PtBi}_{16}$ in the ordered and disordered NiAs structures. Moments on $\mathrm{Pt}$ and $\mathrm{Bi}$ are of the order of $0.13 \mu_{B}$ and $-0.17 \mu_{B}$, respectively.

\begin{tabular}{lccc}
\hline \hline Structure & $\begin{array}{c}\text { Ordered } \\
\text { NiAs supercell }\end{array}$ & $\begin{array}{c}\text { Disordered } \\
\text { NiAs supercell }\end{array}$ & $\begin{array}{c}\text { Interstitial } \\
\mathrm{Mn}\end{array}$ \\
\hline $\mathrm{Mn}_{16} \mathrm{Bi}_{16}$ & 3.66 & 3.18 & -3.10 \\
$\mathrm{Mn}_{15} \mathrm{PtBi}_{16}$ & 3.34 & 2.84 & -3.20 \\
\hline \hline
\end{tabular}

atom per supercell from the regular site to the interstitial site $\left(\mathrm{Mn}_{15} \mathrm{PtBi}_{16}\right)$.

The calculations predict AFM couplings between the interstitial and regular $\mathrm{Mn}$ atoms in both (ii) disordered $\mathrm{MnBi}$ and (iv) disordered MnBi-Pt. However, the magnitude of $J^{\prime}=\left|E_{\mathrm{AFM}}-E_{\mathrm{FM}}\right| / 2$ is larger for $\mathrm{Mn}_{16} \mathrm{Bi}_{16}(152.5 \mathrm{meV})$ than for $\mathrm{Mn}_{15} \mathrm{PtBi}_{16}(27.5 \mathrm{meV})$. The self-doping energy necessary to move a Mn atom onto an interstitial site depends on the stoichiometry and on whether one assumes AFM or FM coupling. In the physically realized AFM case, the Mn self-doping energy decreases from $0.71 \mathrm{eV}\left(\mathrm{Mn}_{16} \mathrm{Bi}_{16}\right)$ to $0.60 \mathrm{eV}\left(\mathrm{Mn}_{15} \mathrm{PtBi}_{16}\right)$. These results show that doping $\mathrm{MnBi}$ with $\mathrm{Pt}$ facilitates the displacement of $\mathrm{Mn}$ atoms from their regular sites to the interstitial sites. Table I summarizes the calculated magnetic moments for $\mathrm{Mn}_{16} \mathrm{Bi}_{16}$ and $\mathrm{Mn}_{15} \mathrm{PtBi}_{16}$. The main reason for the calculated moment reduction due to $\mathrm{Pt}$ is the spin reversal on the interstitial site.

$\mathrm{Mn}_{15} \mathrm{PtBi}_{16}$ has a nominal Pt content of $3.125 \%$ and can therefore be compared with our $x=3$ sample. Experimentally, the addition of $3 \% \mathrm{Pt}$ reduces the average magnetization by about $28 \%$. Assuming Pt displaces the Mn atoms onto interstitial sites, the first-principles calculations yield a reduction of about $20 \%$. Both reductions are much larger than a simple dilution effect, which would yield a moment decrease of $5.5 \%$, as shown in Fig. 2(b). The difference between experiment (28\% reduction) and theory (20\% reduction) probably reflects the simplicity of the structural model used in the first-principles calculations. In particular, the Pt may strongly reduce locally the Mn-Mn exchange of atoms on regular lattice sites, causing local noncollinear Mn moments.

We next investigate how Pt affects the transport behavior of the alloy. The temperature dependence of the resistivity $\rho=\rho_{x x}$ of the $\mathrm{Mn}_{55_{-}} \mathrm{Pt}_{x} \mathrm{Bi}_{45}$ films from 2 to $300 \mathrm{~K}$ shows that the films are metallic but the resistivities of the Pt-doped films are higher than that of undoped MnBi. The resistance ratio $\rho(300 \mathrm{~K}) / \rho(10 \mathrm{~K})$ decreases almost linearly as the $\mathrm{Pt}$ concentration $\left(c_{\mathrm{Pt}}\right)$ increases from $0 \%$ to $4.5 \%$, indicating that the Pt impurity scattering yields a substantial contribution to the residual resistivity. However, this does not preclude the Kondo-type scattering of the conduction electrons by loosely coupled Mn atoms.

Unexpected features of the resistivity of our MnBi:Pt are the low-temperature resistance minimum of the doped films and a positive magnetoresistance. Resistance minima have been found in magnetic semiconductors and glasses, but these materials are either dilute or have a low ordering temperature. ${ }^{22-24}$ As shown in Fig. 3(a), the low-temperature $(<10 \mathrm{~K})$ resistivities of our samples with $0 \%$ and $1.5 \%$ 


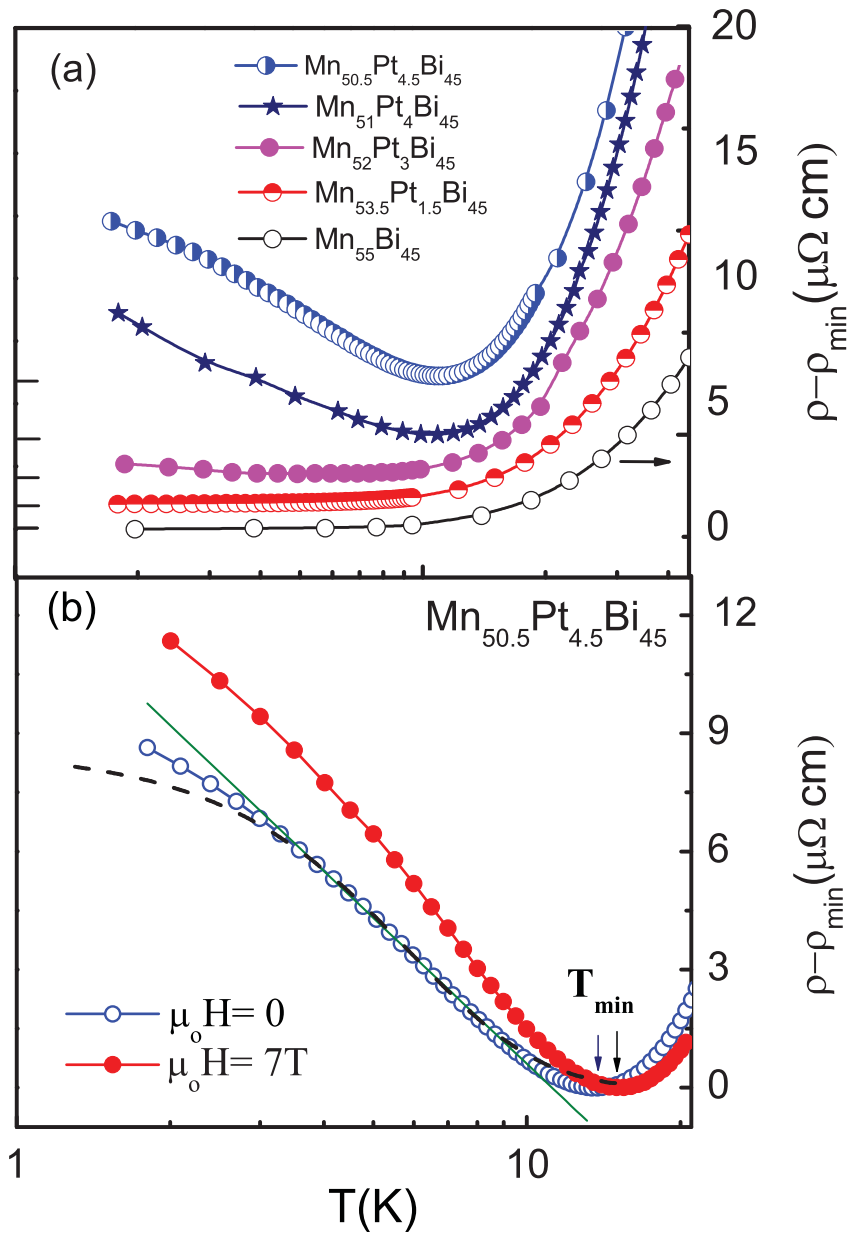

FIG. 3. (Color online) (a) Low-temperature resistivities of Ptdoped $\mathrm{MnBi}$ thin films with Pt concentrations of $0 \%, 1.5 \%, 3 \%$, $4 \%$, and $4.5 \%$. The temperature is on a logarithmic scale and the baselines of the plots for $1.5 \%, 3 \%, 4 \%$, and $4.5 \%$ Pt concentrations are shifted for clarity (see tic marks on the left). The curves are merely guides to the eye. (b) Comparison of the resistivity of $\mathrm{Mn}_{50.5} \mathrm{Pt}_{4.5} \mathrm{Bi}_{45}$ film in zero magnetic field (open circles) and in a field of $7 \mathrm{~T}$ (solid circles). The straight-line fit to the resistivity below $T_{\min }$ shows that the temperature dependence of the resistivity is logarithmic. The dashed black line fits the empirical form of the numerical renormalization group (NRG) results $\rho_{m}(T)=\rho_{0}\left[1+\left(2^{1 / \alpha_{s}}-1\right)\left(T / T_{k}\right)^{\xi_{s}}\right]^{-\alpha_{s}}$ to the low-temperature resistivity data.

Pt remain almost constant at $12 \mu \Omega \mathrm{cm}$ and $21 \mu \Omega \mathrm{cm}$, respectively. However, the samples with higher Pt concentrations $(3 \%, 4 \%$, and $4.5 \%)$ exhibit resistivity minima with an approximate logarithmic temperature dependence below the minimum. Below $3 \mathrm{~K}$, the resistivity of $4.5 \%$ Pt-doped film deviates from the logarithmic behavior and shows a sign of saturation which is consistent with the unitary limit of Kondo scattering at very low temperature ${ }^{9,10}$ [see Fig. 3(b)]. To check whether the increase in resistivity due to magnetic impurity scattering of conduction electrons is consistent with the numerical renormalization group (NRG) results, ${ }^{25,26}$ we fitted our data to the empirical form of NRG results, $\rho_{m}(T)=\rho_{0}\left[1+\left(2^{1 / \alpha_{s}}-1\right)\left(T / T_{k}\right)^{\xi_{s}}\right]^{-\alpha_{s}}$, where $\xi_{s}$ and $\alpha_{s}$ are the fitting parameters and $\rho_{0}$ is the residual resistivity. As shown in Fig. 3(b), the data agree moderately with the

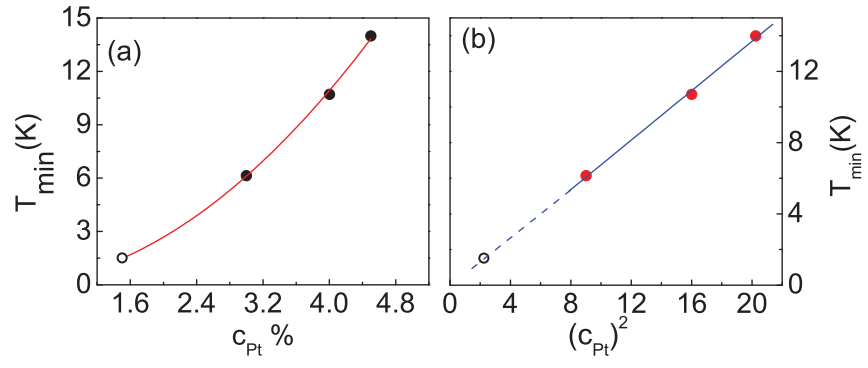

FIG. 4. (Color online) (a) Temperature of resistivity minima $\left(T_{\min }\right)$ as a function of $\mathrm{Pt}$ concentration $c_{\mathrm{Pt}}$ in a linear scale. (b) Extrapolation of the data in $T_{\min } \mathrm{vs} c_{\mathrm{Pt}}{ }^{2}$ plot to estimate the value of $T_{\min }$ for $1.5 \% \mathrm{Pt}$. The open circle corresponds to the estimated value of $1.5 \mathrm{~K}$ for $1.5 \% \mathrm{Pt}$ concentration.

NRG results for $\xi_{s}=2$ and Kondo temperature $T_{k}=5.1 \mathrm{~K}$, where the experimental data fall above the NRG results at low temperature. This suggests that the magnetic moments responsible for Kondo scattering are not fully screened at our lowest available temperature. This is consistent with the fact that the experimental $\rho(T)$ curve shows a slow approach to saturation with decreasing temperature. We also have found that the temperature $T_{\min }$ of the resistivity minimum increases according to $T_{\min } \propto c_{\mathrm{Pt}}^{2}$ from $6 \mathrm{~K}$ for $c_{\mathrm{Pt}}=3 \%$ to $14 \mathrm{~K}$ for $c_{\mathrm{Pt}}=4.5 \%$, as shown in Figs. 4(a) and 4(b). The value of $T_{\min }$ for $1.5 \% \mathrm{Pt}$ estimated from the extrapolation of the $T_{\min }$ versus $c_{\mathrm{Pt}}^{2}$ plot is $1.5 \mathrm{~K}$, which is consistent with the fact that the sample with $1.5 \% \mathrm{Pt}$ doping does not show a resistivity minimum down to our lowest available temperature of $2 \mathrm{~K}$ [see Fig. 4(b)]. We note that $T_{\min }$ does not follow the standard scaling law with Pt concentration. This is natural because the Kondo scattering is not realized by the added Pt atoms but by the concentration of loosely exchange-coupled interstitial Mn atoms. The Pt changes this concentration as a regular but nonlinear function of the Pt content.

Figure 3(b) compares the resistivity of $\mathrm{Mn}_{50.5} \mathrm{Pt}_{4.5} \mathrm{Bi}_{45}$ in zero magnetic field and in a perpendicular magnetic field of $7 \mathrm{~T}$. The measured magnetoresistance is positive, as contrasted to the usual suppression of the Kondo effect by an external magnetic field, which corresponds to a negative magnetoresistance. Although a positive magnetoresistance is observed in simple metals ${ }^{27}$ and also in some ferromagnets due to a quantum interference effect, ${ }^{28}$ we propose a different model to explain the resistance minimum and the unexpected positive magnetoresistance in our system. We assume as discussed above that $\mathrm{Pt}$ displaces some of the $\mathrm{Mn}$ atoms from their regular positions in the NiAs structure to interstitial sites. This displacement and the presence of the Pt atoms weaken the ferromagnetic exchange between the Mn atoms and eventually create a certain fraction of interstitial Mn spins that are only weakly exchange-coupled to the remainder of the crystal.

The above first-principles calculations yield the exchange constants $J^{\prime}=\left|E_{\mathrm{AFM}}-E_{\mathrm{FM}}\right| / 2$ for a Mn atom displaced to an interstitial site, namely $J^{\prime} / k_{B}=-1770 \mathrm{~K}$ for the $\mathrm{Mn}_{16} \mathrm{Bi}_{16}$ supercell and $J^{\prime} / k_{B}=-320 \mathrm{~K}$ for the $\mathrm{Mn}_{15} \mathrm{PtBi}_{16}$ supercell. This must be contrasted to the ferromagnetic exchange of the $\mathrm{Mn}$ atoms on their regular lattice sites $\left(J_{o}>0\right)$. In fact, there are many ways to coordinate an interstitial $\mathrm{Mn}$ atom by $\mathrm{Mn}, \mathrm{Bi}$, and Pt nearest and more distant neighbors, and 


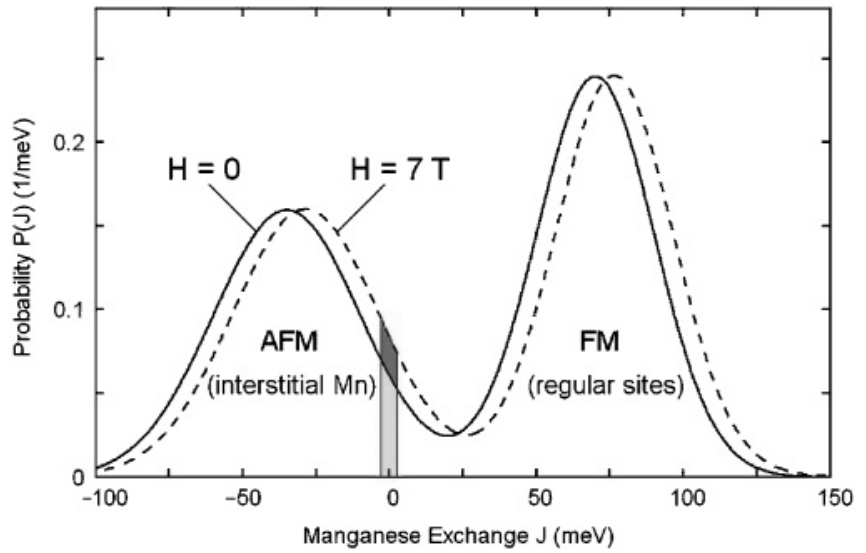

FIG. 5. Schematic distribution of Mn exchange constants in Pt-doped MnBi. Uncoupled Mn spins (gray area) undergo Kondo flipping, and a magnetic field shifts the distribution toward positive exchange fields. The Pt affects both peaks and reduces the magnitude of the AFM exchange field acting on interstitial Mn.

large supercells would be necessary to accurately describe the exchange interaction of the individual Mn atoms. However, we have calculated the effect of the larger Pt concentration. This allows us to explore the effect of two Pt atoms in the neighborhood of an interstitial $\mathrm{Mn}$. We find that the presence of a second $\mathrm{Pt}$ as the nearest neighbor alters the exchange interactions and, depending on the relative position of the second Pt atom with respect to the first, the onsite exchange parameter may be either FM $(0.022 \mathrm{eV}=260 \mathrm{~K})$ or $\mathrm{AFM}$ $(-0.034 \mathrm{eV}=-396 \mathrm{~K})$ with a reduced magnitude of the exchange.

Figure 5 illustrates this mechanism by showing a schematic distribution $P(J)$ of the Mn exchange interactions. The Kondo effect is realized by $\mathrm{Mn}$ atoms with weak interatomic exchange (gray area). These spins are able to undergo Kondo spin-flip scattering and to yield a resistance minimum. To understand the positive magnetoresistance, it is necessary to consider a distribution $P(J)$ of the Mn exchange interaction. The FM coupling of the Mn on the regular sites $\left(J_{o}>0\right)$ and the AFM coupling of $\mathrm{Mn}$ of the lattice sites considered above $\left(J^{\prime}<\right.$ 0 ) indicate broad exchange distributions with two or more smoothed FM and AFM peaks. As a consequence, $P(J)$ is nonzero over a fairly broad range of exchange constants $J$. If some of these $J$ values are zero or very small, then they can undergo Kondo flipping and enhance the resistivity. The challenge is now to estimate the fraction $W$ of such spins as a function of the magnetic field $H$.

The Kondo effect persists down to very low concentrations of localized moments, and it is safe to assume that $W \ll 1$. In fact, most Mn spins are subject to strong AFM or FM interactions, and neither the external magnetic field nor the Kondo interactions are able to switch these spins. This means that most Mn spins are frozen (Fig. 5) and that the remaining fraction $W$ of loosely coupled spins $\hat{s}_{i}$ interact with the remaining frozen spins $\hat{s}_{j^{\prime}}$ via a mean-field interaction of the type

$$
\hat{H}=-\sum_{j} J_{i j} \hat{s}_{i} \cdot\left\langle\hat{s}_{j^{\prime}}\right\rangle-\mu_{0} \mu \mathbf{H} \cdot \sum \hat{\mathbf{s}}_{\mathbf{i}} .
$$

This equation, which neglects the interactions between loose spins, provides a definition for the effective exchange of the $i$ th spin, $J(i)=\sum_{j} J_{i j}\left\langle\hat{s}_{j z^{\prime}}\right\rangle$. The fraction $W$ of loose spins is then described by the distribution $P(J)$. We note that $W$ is a largely unknown function of the Pt concentration. In fact, the smearing of the main peaks of the distribution function in Fig. 5 requires a certain degree of disorder, and virtually no Kondo scattering is expected for small Pt concentrations. This is consistent with the aforementioned $c_{\mathrm{Pt}}{ }^{2}$ dependence of $T_{\min }$.

Equation (1) means that the magnetic field adds to the exchange and effectively shifts the distribution $P(J)$. A trivial example is that a strong positive field fixes all spins in the $\uparrow$ direction and completely suppresses the Kondo effect. However, our fields are rather weak, of the order of $5 \mathrm{~K}$ in temperature units, compared to the FM or AFM exchange interaction of the order of $500 \mathrm{~K}$. The probability $W$ therefore corresponds to a thin slice of the distribution, as indicated in Fig. 5. Assuming that Kondo flipping occurs for some range $|J| \leqslant \delta J$, we find

$$
W=\int_{-\delta J}^{\delta J} P\left(J-\mu_{0} \mu H\right) d J .
$$

With increasing field $H$, the fraction $W$ of loose spins increases or decreases, depending on $P(J)$, and the corresponding change is described by $W=2 \delta J P-2 \delta J \mu_{0} \mu H d P / d J$. Here both $P$ and $d P / d J$ refer to $H=0$. Since the magnetoresistance increases with $W$, negative and positive slopes $d P / d J$ correspond to a positive or negative magnetoresistance, respectively. For the schematic exchange distribution of Fig. 5, the magnetoresistance is positive (dark gray area), which provides a qualitative explanation of positive magnetoresistance. In particular, our first-principles calculations indicate that the addition of $\mathrm{Pt}$ reduces the magnitude of the negative exchange of the interstitial Mn. This means that the AFM peak of the distribution $P(J)$ moves toward $J=0$, thereby enhancing $P$ and $d P / d J$ compared to the undoped material.

\section{CONCLUSION}

In summary, our MnBi-Pt system shows three unusual features. First, it exhibits a low-temperature Kondo resistance minimum in a strong ferromagnetic material with a Curie temperature well above room temperature. Second, we have shown that the nonmagnetic Pt content controls the Mn spin correlations and the Kondo effect. Third, the system exhibits a positive magnetoresistance, which is unusual for Kondo systems. Both the Kondo effect and the positive magnetoresistance are explained as a materials-specific exchange effect, caused by the disorder in the MnBi lattice due to the Pt-induced displacement of $\mathrm{Mn}$ atoms onto interstitial sites.

\section{ACKNOWLEDGMENTS}

We would like to thank R. D. Kirby and E. Tsymbal for helpful comments. This work was supported by the NSF-MRSEC, DOE (P. K. and D. J. S.) and NCMN. The computational part was performed utilizing the Blackforest Cluster Computing Facility at UNO. 
${ }^{1}$ J. Kondo, Prog. Theor. Phys. 32, 37 (1964).

${ }^{2}$ W. Meissner and B. Voigt, Ann. Phys. 399, 761 (1930).

${ }^{3}$ J. Martinek, M. Sindel, L. Borda, J. Barnas, J. König, G. Schön, and J. von Delft, Phys. Rev. Lett. 91, 247202 (2003).

${ }^{4}$ A.-D. Zhao, Q.-X. Li, L. Chen, H.-J. Xiang, W.-H. Wang, Sh. Pan, B. Wang, X.-D. Xiao, J.-L. Yang, J. G. Hou, and Q.-Sh. Zhu, Science 309, 1542 (2005).

${ }^{5}$ M. R. Calvo, J. Fernández-Rossier, J. J. Palacios, D. Jacob, D. Natelson, and C. Untiedt, Nature (London) 458, 1150 (2009).

${ }^{6}$ J. Kondo, Proc. Jpn. Acad. Ser. B 82, 328 (2006).

${ }^{7}$ N. B. Brandt and V. V. Moschalkov, Adv. Phys. 33, 373 (1984).

${ }^{8}$ S. Doniach, Physica B+C 91, 231 (1977).

${ }^{9} \mathrm{P}$. Fulde, Electron Correlations in Molecules and Solids (Springer, Berlin, 1991).

${ }^{10}$ A. C. Hewson, The Kondo Problem to Heavy Fermions (Cambridge University, London, 1993).

${ }^{11}$ R. Skomski and J. M. D. Coey, Permanent Magnetism (Institute of Physics, Bristol, 1999).

${ }^{12}$ G. Q. Di, S. Iwata, S. Tsunashima, and S. Uchiyama, J. Magn. Magn. Mater. 104, 1023 (1992).

${ }^{13}$ R. F. Sabiryanov and S. S. Jaswal, Phys. Rev. B 53, 313 (1996).

${ }^{14}$ R. R. Heikes, Phys. Rev. 99, 446 (1955).

${ }^{15}$ U. Rüdiger and G. Güntherodt, J. Appl. Phys. 88, 4221 (2000).

${ }^{16}$ J. B. Yang, W. B. Yelon, W. J. James, Q. Cai, M. Kornecki, S. Roy, N. Ali, and Ph. l'Heritier, J. Phys. Condens. Matter 14, 6509 (2002).
${ }^{17} \mathrm{~J}$. B. Goodenough, Magnetism and the Chemical Bond (Wiley, New York, 1963).

${ }^{18}$ T. Chen and W. E. Stutius, IEEE Trans. Magn. 10, 581 (1974).

${ }^{19}$ P. Kharel, R. Skomski, R. D. Kirby, and D. J. Sellmyer, J. Appl. Phys. 107, 09E303 (2010).

${ }^{20}$ B. W. Roberts, Phys. Rev. 104, 607 (1956).

${ }^{21}$ J. P. Perdew, K. Burke, and M. Ernzerhof, Phys. Rev. Lett. 77, 3865 (1996).

${ }^{22}$ R. Hasegawa and C. C. Tsuei, Phys. Rev. B 3, 214 (1971).

${ }^{23}$ G. S. Grest and S. R. Nagel, Phys. Rev. B 19, 3571 (1979).

${ }^{24}$ H. T. He, C. L. Yang, W. K. Ge, J. N. Wang, X. Dai, and Y. Q. Wang, Appl. Phys. Lett. 87, 162506 (2005).

${ }^{25}$ J. J. Parks, A. R. Champagne, T. A. Costi, W. W. Shum, A. N. Pasupathy, E. Neuscamman, S. Flores-Torres, P. S. Cornaglia, A. A. Aligia, C. A. Balseiro, G. K.-L. Chan, H. D. Abruña, and D. C. Ralph, Science 328, 1370 (2010).

${ }^{26}$ T. A. Costi, L. Bergqvist, A. Weichselbaum, J. von Delft, T. Micklitz, A. Rosch, P. Mavropoulos, P. H. Dederichs, F. Mallet, L. Saminadayar, and C. Bäuerle, Phys. Rev. Lett. 102, 056802 (2009).

${ }^{27}$ A. Campbell and A. Fert, Handbook of Magnetic Materials (Wohlfarth, North-Holland, Amsterdam, 1982).

${ }^{28}$ M. Ziese, Phys. Rev. B 68, 132411 (2003). 